\title{
Intraoperative prediction of the two axillary lymph node macrometastases threshold in patients with breast cancer using a one-step nucleic acid cytokeratin-19 amplification assay
}

\author{
VICTORIA FUNG $^{1}$, STAN KOHLHARDT ${ }^{1}$, PATRICIA VERGANI ${ }^{2}$, \\ GREGORY J. ZARDIN ${ }^{2}$ and NORMAN R. WILLIAMS ${ }^{3}$ \\ ${ }^{1}$ Department of Breast and Plastic Surgery, Sheffield Breast Center; ${ }^{2}$ Department of \\ Histopathology, Royal Hallamshire Hospital, S10 2JF Sheffield; ${ }^{3}$ Division of Surgery and \\ Interventional Science, University College London, WC1E 6AU London, UK
}

Received March 30, 2017; Accepted July 11, 2017

DOI: $10.3892 / \mathrm{mco} .2017 .1404$

\begin{abstract}
The aim of the present study was to assess the sensitivity, specificity and practicality of using a one-step nucleic acid amplification (OSNA) assay during breast cancer staging surgery to predict and discriminate between at least 2 involved nodes and more than 2 involved nodes and facilitate the decision to provide axillary conservation in the presence of a low total axillary node tumour burden. A total of 700 consecutive patients, not treated with neo-adjuvant chemotherapy, received intraoperative sentinel lymph node (SLN) analysis using OSNA for cT1-T3 cN0 invasive breast cancer. Patients with at least one macrometastasis on whole-node SLN analysis underwent axillary lymph node dissection (ALND). The total tumour load (TTL) of the macrometastatic SLN sample was compared with the non-sentinel lymph node (NSLN) status of the ALND specimen using routine histological assessment. In total, $122 / 683$ patients (17.9\%) were found to have an OSNA TTL indicative of macrometastasis. In addition, 45/122 (37\%) patients had NSLN metastases on ALND with a total positive lymph node burden exceeding the American College of Surgeons Oncology Group Z0011 trial threshold of two macrometastatic nodes. The TTL negative predictive value was 0.975 [95\% confidence interval (CI), 0.962-0.988]. The area under the curve for the receiver operating characteristic curve was 0.86 (95\% CI, 0.81-0.91), indicating that SLN TTL was associated with the prediction (and partitioning) of total axillary disease burden. OSNA identifies a TTL threshold value where, in the presence of involved SLNs, ALND may
\end{abstract}

Correspondence to: Dr Stan Kohlhardt, Department of Breast and Plastic Surgery, Sheffield Breast Center, Royal Hallamshire Hospital, 4 Claremont Place, S10 2JF Sheffield, UK

E-mail: stan.kohlhardt@sth.nhs.uk

Key words: breast cancer, sentinel lymph node, axillary lymph node dissection, one-step nucleic acid amplification assay, total tumour load, cytokeratin-19 be avoided. This technique offers objective confidence in adopting conservative management of the axilla in patients with SLN macrometastases.

\section{Introduction}

Sentinel lymph node biopsy (SLNB) has replaced complete axillary lymph node dissection (ALND) as the current standard of care for axillary node staging in patients with clinically node-negative breast cancer (1). When the sentinel nodes are found to be free from metastatic disease, no further axillary treatment is recommended (1). The role of ALND, for patients with positive sentinel lymph nodes, is currently being redefined. Research has indicated that patients with sentinel lymph node (SLN) micrometastases treated with SLNB alone have similar disease-free and overall survival to those receiving ALND (2). This has also been observed for stage T1-2 disease with $\leq 2$ macro-metastatic SLNs treated with breast conservation surgery, whole-breast radiotherapy and adjuvant systemic therapy (3). Therefore, ALND may be over-treatment for patients with early breast cancer and a low burden of axillary node involvement.

A reliable intraoperative technique that predicts non-sentinel lymph node (NSLN) involvement would offer selective and more conservative treatment of the axilla in a single surgical procedure. This would avoid unnecessary surgery and its associated morbidity while providing benefits to the patient, conserving resources and complying with emerging clinical practice guidelines (4).

SLN assessment with one-step nucleic acid amplification (OSNA) provides an intraoperative molecular-based objective whole-node assessment of SLN disease burden that is independent of the size or number of lymph nodes tested (5). For these reasons, OSNA has greater potential to predict NSLN involvement than routine histopathology assessment, which cannot offer timely intraoperative SLN evaluation, remains subjective, categorical, and is exposed to sampling errors with sub-total node assessment (6,7). OSNA amplifies cytokeratin-19 (CK19) mRNA in SLN samples, typically providing a quantitative measurement of metastatic disease burden in $35 \mathrm{~min}$. The total 
CK19 mRNA copy number of a SLN biopsy (total tumour load, TTL) may predict NSLN involvement and axillary node disease burden, facilitating the decision to proceed with, or avoid, complete ALND.

The present study compared SLN OSNA TTL with NSLN involvement following ALND, and assessed the sensitivity and specificity of TTL, and patient and tumour characteristics to predict NSLN metastatic burden. The present findings have generated a selective treatment protocol for the conservative management of the axilla in patients with early breast cancer that may be used in the pre- and intraoperative setting.

\section{Patients and methods}

Patients. The present study was a retrospective, single-centre cohort study of patients diagnosed with breast cancer from symptomatic and screening services. A total of 700 consecutive patients (681 females and 3 males; mean age, 62 years; range, 23-93 years) treated between December 2012 and August 2015 at the Royal Hallamshire Hospital (Sheffield, UK) who underwent a successful SLN biopsy with OSNA assessment were studied. The patients had primary invasive cT1-3 breast carcinoma, a clinically negative axilla, normal pre-operative axillary ultrasound, or benign ultrasound-guided axillary node biopsy, and were medically fit for general anaesthetic and axillary treatment. Patients with prior neo-adjuvant chemotherapy, ipsilateral axillary surgery, recurrent disease or extensive ductal carcinoma in situ (DCIS) without invasion were excluded. Subset analysis was performed for patients who had metastatic axillary disease identified by OSNA and subsequent ALND. The following parameters were recorded: Age, tumour size and grade, multifocality, histological subtype, type of surgery, oestrogen receptor status, human epidermal growth factor receptor 2 (HER2) status, Ki67, the presence of lymphovascular invasion, total number of SLNs and NSLNs, the number of positive and negative SLNs and NSLNs, and ratio of the number of positive SLNs to the total number of removed SLNs and NSLNs following ALND. CK19 mRNA copy number (copies $/ \mu 1$ ) in each SLN were recorded. CK19 expression was not routinely tested on pre-operative biopsy specimens. For statistical analysis, when $\geq 2$ SLNs were involved, the combined value of CK19 mRNA copies was calculated. The TTL was defined as the total CK19 mRNA copy number in the positive SLNs (with units of copies $/ \mu 1$ ). The TTL of the macrometastatic SLN sample was compared with the total lymph node status and NSLN status of ALND, following routine histological assessment with haematoxylin and eosin staining (8).

SLN identification. SLNs were identified using a standard protocol of combination radiopharmaceutical and blue dye, as described by Mansel et al (9). ${ }^{99 \mathrm{~m}} \mathrm{Tc}-$ labelled albumin nanocolloid (Nanocoll ${ }^{\circledR}$; GE Healthcare Life Sciences, Little Chalfont, UK) was injected intradermally $(0.1-0.5 \mathrm{ml})$ at a single periareolar site corresponding to the tumour quadrant; $40 \mathrm{MBq}$ the day before surgery or $20 \mathrm{MBq}$ on the day of surgery. Patent Blue V dye (Laboratoire Guebet, Aulnay-sous-Bois, France), $2 \mathrm{ml}$ undiluted, was injected subdermally at a single periareolar site corresponding to the tumour quadrant immediately prior to surgery. Under general anaesthetic, SLNs were identified and removed prior to breast tumour excision, and sent on ice to the Pathology Department. No more than two nodes were sent for assessment by OSNA. Any additional SLNs were sent for routine fixation, hematoxylin and eosin staining (8) and delayed reporting. Therapeutic local excision, therapeutic mammoplasty or mastectomy was performed as part of the planned breast cancer treatment. Each SLN, trimmed of fat, was weighed and recorded. SLNs weighing $<50 \mathrm{mg}$ were too small to be processed by OSNA, and were therefore diverted to routine histological assessment. SLNs weighing $>600 \mathrm{mg}$ were divided into two or more pieces and processed separately, and the results combined.

OSNA. The OSNA assay (Sysmex Europe GmbH, Norderstedt, Germany) was performed according to the manufacturer's protocols, as described by Tsujimoto et al (5). Each SLN was homogenized in $4 \mathrm{ml}$ homogenizing buffer on ice. The lysate was centrifuged to remove fat, cellular debris and other contaminants and the mRNA containing supernatant was extracted and diluted. A 2- $\mu 1$ aliquot of the buffered lymph node lysate was used for automated real-time amplification of CK19 mRNA via reverse transcription loop-mediated isothermal amplification with a ready-to-use reagent kit on the RD-100i (Sysmex Europe GmbH). The rate of amplification was measured spectrophotometrically and the CK19 copy number was calculated by comparison to a standard curve. Based on the number of CK19 mRNA copies/ $\mu 1$, the result was assessed in accordance with the cut-off levels determined in a study by Tsujimoto et al (5), with macrometastasis (OSNA ++) defined as $>5,000$ copies $/ \mu 1$ of CK19 mRNA, micrometastasis (OSNA + ) as 250-5,000 copies/ $\mu 1$ and non-metastasis (OSNA -) as $<250$ copies/ $\mu 1$. The OSNA results were immediately communicated by telephone to the surgeon within $45 \mathrm{~min}$ of sample receipt. Patients with at least one macrometastasis on intraoperative OSNA analysis underwent levels I, II and III ALND. Between December 2012 and June 2013, a positive OSNA result for one or two nodes with micro-metastases also triggered an immediate ALND. In June 2013, the departmental protocol was amended to recommend the removal of two further nodes for routine histological processing, with a delayed ALND if these returned macrometastatic involvement.

The remaining lymph nodes not involved in the OSNA test were processed according to the UK Breast Cancer pathology protocol (8). Lymph nodes $<5 \mathrm{~mm}$ were bisected whereas larger nodes were sliced at 3-mm intervals and single sections assessed using haematoxylin and eosin staining. Immunohistochemical staining was not used for evaluation of NSLNs.

Preoperative assessment of axilla. Patients underwent axillary ultrasonography (US) at the time of breast assessment, or soon after the diagnosis of breast carcinoma. Those with abnormal lymph node morphology according to local protocol (cortical thickening 2-3 mm, focal bulge, rounded shape, partial or complete loss of fatty hilum, non-hilar blood flow, or partial/complete replacement of node with mass) underwent US-guided lymph node biopsy. Patients with confirmed invasive disease on lymph node biopsy proceeded to neo-adjuvant therapy or ALND without SLNB.

Patient data were anonymised, and collected retrospectively, without influence on patient therapy. The ethical considerations of the present study were approved by the 
Clinical Effectiveness Unit of the Sheffield Teaching Hospitals NHS Trust (Sheffield, UK).

Statistical analysis. Distributions of continuous variables were determined by visual inspection of frequency-distribution plots; variables were summarised as the mean and confidence interval (after transformation if required), or median and interquartile range, as appropriate. Tests for association between categorical variables were determined using the Chi-square and Fisher's exact tests. $\mathrm{P}<0.05$ was considered to indicate a statistically significant difference. Receiver operating characteristic (ROC) analyses were performed to compute the area under the curve (AUC) to estimate concordance. The statistical analysis was conducted using SAS software, version 9.4 (SAS Institute, Inc., Cary, NC, USA) for Windows 7.

\section{Results}

Clinical cohort. A total of 1,315 patients were diagnosed with breast carcinoma between December 2012 and August 2015 at the institution (Fig. 1). Of these, 700 consecutive patients received OSNA during surgery for a preoperatively identified cT1-T3 cN0 breast carcinoma. Sixteen cases were excluded from the present study; 15 patients had extensive DCIS only and 1 patient had received incomplete neo-adjuvant therapy. There were 681 female patients and 3 male cases (mean age, 62 years; range, 23-93 years) in the remaining study cohort. Of the cases, 9 were bilateral.

In contrast to the American College of Surgeons Oncology Group (ACOSOG) Z0011 study (3), all the patients in our unit underwent axillary US prior to breast surgery. Those with abnormal lymph node morphology underwent immediate US-guided biopsy, and those with confirmed metastatic disease were recommended to undergo ALND without SLNB/OSNA, which was the protocol at the time of the present study. These assessments removed $14.5 \%(191 / 1315)$ of our total new diagnosis patient cohort from analysis. These patients did not receive further assessment to explore the role of conservative management of the axilla as part of their treatment.

Clinicopathological characteristics. The mean number of nodes harvested for OSNA per patient was 1.94, with a total of 1,356 SLNs assessed. A total of 123/684 patients (17.9\%) were found to have OSNA CK19 mRNA copy numbers indicative of macro-metastasis and all but 1 patient underwent ALND. In total, 45/122 (37\%) patients had NSLN metastases on ALND with a total positive lymph node burden exceeding the ACOSOG Z0011 threshold of two macro-metastatic nodes (Fig. 2).

The distribution of tumour sizes within the cohort displayed the expected log-normal distribution for total and sub-group distribution of size by node involvement. There were 143 tumours with a diameter $\leq 10 \mathrm{~mm}$, and none were associated with $>2$ macrometastases in ALND. TTL was the only clinicopathological variable significantly associated with risk of NSLN involvement and three or more positive nodes $(\mathrm{P}<0.0001)$. Tumour size $(\mathrm{P}=0.14)$, tumour grade $(\mathrm{P}=0.84)$, oestrogen receptor status $(\mathrm{P}=0.09)$, HER2 $(\mathrm{P}=1.00)$ and lymphovascular invasion $(\mathrm{P}=0.30)$ were not significantly associated with the risk of NSLN involvement and three or more positive nodes (data not shown).
TTL, axillary lymph node burden and prediction. Sensitivity and specificity of OSNA TTL vs. NSLN status using the protocol thresholds of TTL $\geq 250, \geq 5000$ and $\geq 15,000$ copies $/ \mu 1$ are detailed in Table I. Diagnostic accuracy of TTL, as demonstrated by ROC AUC, was 0.86 (Fig. 3).

In the present cohort, $11.4 \%(13 / 114)$ of the grade 1 tumours had evidence of SLN or NSLN metastases, compared with $19.9 \%(70 / 351)$ of the grade 2 and $19.4 \%$ (42/216) of the grade 3 tumours. The maximum total axillary burden for any grade 1 tumour was four nodes for an 18-mm tumour.

SLNB TTL vs. total lymph node involvement for the entire cohort, and for the subset of patients undergoing breast conserving surgery (BCS), was plotted (Figs. 4 and 5, respectively). The distribution of NSLN metastases was partitioned vertically by the point separation of data found at 15,000 copies $/ \mu 1$ and horizontally by the two lymph node involvement threshold by which the ACOSOG Z0011 trial would not recommend ALND (3). BCS was performed in $499 / 700(71.2 \%)$ patients and they represented a surrogate sub-group for comparison with the ACOSOG Z0011 (3) trial. However, the present group had broader inclusion criteria and was selection-modified by preoperative axillary US filtering. In total, 66/499 patients (13.2\%) had ALND, 27 (41\%) with NSLN involvement and $39(59 \%)$ without NSLN involvement. In total, 20/23 (87\%) patients with TTL $>15,000$ copies/ $\mu 1$ of CK19 mRNA had more than two involved NSLNs on ALND. Sensitivity, specificity, positive predictive value and negative predictive value of SLNB TTL vs. NSLN status using the threshold of TTL $\geq 15,000$ copies/ $\mu$ l for the subset of patients undergoing BCS are detailed in Table I. For patients with a SLNB TTL $<15,000$ copies $/ \mu 1$ and who had $\leq 2$ nodes involved, the NPV was 0.983 . According to Z0011 criteria, 35/499 (7.0\%) patients could be regarded as being over-treated with a $3 / 499$ $(0.6 \%)$ false-negative rate (potential under-treatment).

\section{Discussion}

SLNB remains the standard of care for staging breast carcinoma in the clinically uninvolved axilla (1). Research has suggested that ALND may be safely omitted in patients with a low burden of axillary disease $(3,10,11)$, particularly with micrometastatic disease only (12), but also where only one or two nodes with macrometastases are identified (3). Identifying patients who should proceed with ALND has been attempted with nomograms (13) and prediction models (7) with varying degrees of accuracy. Several studies have reported methods to predict $\geq 4$ lymph node metastases (14-17). However, the majority of these prediction models were constructed using predictors derived from breast resection specimen pathology, such as lymphovascular invasion, and have not been widely adopted as they fail to confidently facilitate one-stage intraoperative decision making about the role of ALND. A small number of studies have reported on the prediction of NSLN metastases in SLN-positive patients, including the use of SLN OSNA TTL $(7,18,19)$.

OSNA was formally approved by the UK National Institute of Clinical Excellence and included in routine surgical practice in 2013 (20). OSNA is at least equally cost effective as routine histology; however, it has substantial patient benefits $(21,22)$. It remains the only intraoperative diagnostic test recommended 


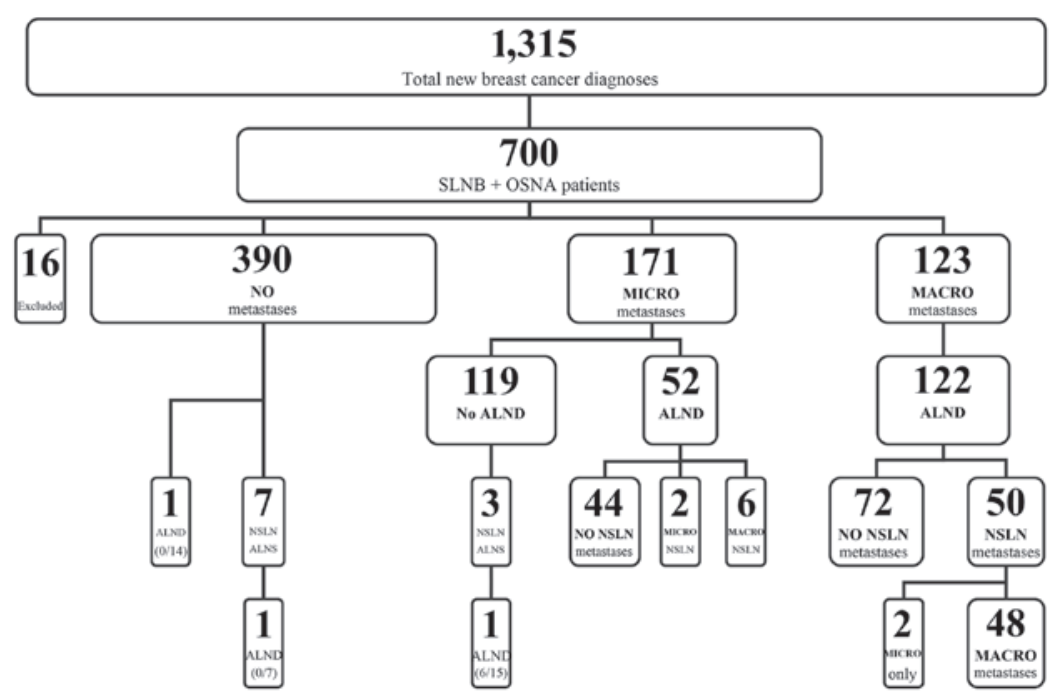

Figure 1. Cohort distribution flow chart. SLNB, sentinel lymph node biopsy; OSNA, one-step nucleic acid amplification; ALND, axillary lymph node dissection; NSLN, non-sentinel lymph node.

for whole-node analysis for detecting SLN metastases during breast surgery in individuals with early invasive breast cancer.

As a categorical diagnostic tool, OSNA alone is insufficient to determine which patients with macro-metastatic nodal disease may be spared ALND. The present study revealed that $60.7 \%$ of the cohort with macrometastatic lymph nodes on SLN OSNA had no further nodal involvement on ALND. Indeed, only $7.9 \%(54 / 683)$ of the entire cohort had more than two nodes with macrometastases. However, the continuous exponential quantification of TTL extends its utility considerably as a predictive tool (7,23-25). Stratification of CK19 mRNA copy numbers facilitates intraoperative decision-making for management of the axilla. In the present cohort of patients, the ROC AUC of 0.86 for two node TTL indicated that SLN TTL represents a good association with the presence of NSLN metastases. However, developing a model that predicts more than two node involvement and NSLN involvement is challenged by the small numbers of patients who satisfy this subset. In total, 21\% of patients in the ALND arm of the Z0011 trial had additional positive nodes (3) whereas this occurred in $37 \%$ of the present cohort, despite our routine use of preoperative axillary US assessment. This observation may reflect a cohort of patients with more favourable disease in the ACOSOG Z0011 study, and/or higher sensitivity of lower disease burden detected with OSNA. Furthermore, the present cohort included 21/683 (3\%) patients with pT3 tumours and the overall mean tumour size was $20 \mathrm{~mm}$ compared to $16 \mathrm{~mm}$ in Z0011 (3). The larger proportion of patients with additional NSLN node involvement in the present cohort may have contributed to the generation of an ROC AUC $>0.8$. Identifying any predictive markers with statistical significance was not possible using traditional clinicopathological factors in the present study. When lymphovascular invasion was reported in the resection specimen, it was twice as likely that NSLN metastases would be present than otherwise. If lymphovascular invasion was reported as being absent, the likelihood of NSLN metastases was $30 \%$ less. These findings, however, did not translate into statistical significance for predicting the risk of additional NSLN disease.

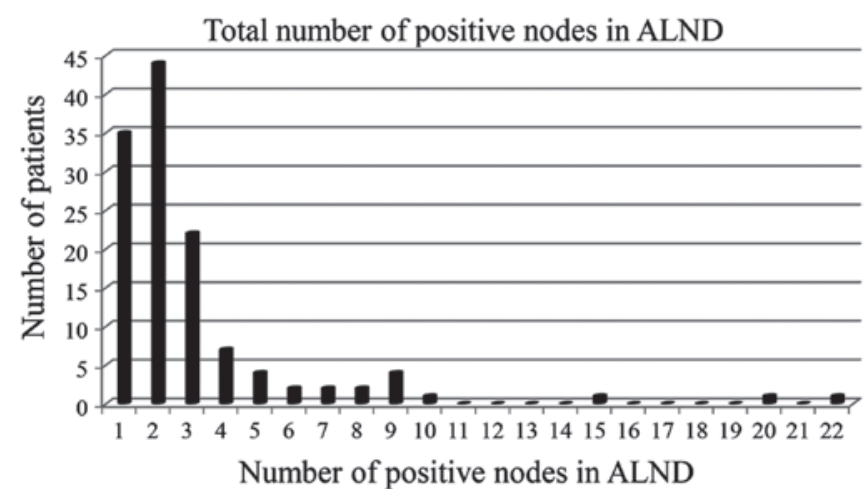

Figure 2. Distribution of the total number of positive nodes in an ALND for 122 patients with macrometastases. ALND, axillary lymph node dissection.

A study by Kubota et al (18) reported a single-centre standardised method for SLN detection that used indocyanine green; whereas, the types of injection and the use of radioisotope, with or without dye, varied according to institutional practice in a multi-centre study by Piñero-Madrona et al (19). The present single-centre dual technique of SLN detection is uniform and standardised (9). Differences in the methods of SLN detection, in particular OSNA TTL quantification restricted to two nodes in the present study, may account for some of the differences observed in ROC AUC values and in the association of NSLN positivity with other putative predictive variables. The mean number of SLNs removed in the studies by Kubota et al (18) and Piñero-Madrona et al (19) are not reported for comparison. An average SLN harvest $>2.0$ may suggest some persistence in retrieval, diluting the residual pool of NSLN with the removal of some nodes that would otherwise be accounted for in a subsequent ALND analysis. This would impact on how the relationship between SLN TTL burden and associated NSLN positivity is interpreted and compared in practice.

The present study identified an extension of the TTL threshold for conservative management of the axilla beyond 5,000 to 15,000 copies $/ \mu 1$ and confirmed the findings of 
Table I. Sensitivities and specificities of OSNA TTL of cytokeratin-19 mRNA copy numbers.

A, OSNA by NSLN using TTL cut-off of $250^{\mathrm{a}}$ copies/ $\mu 1$ for all the patients

\begin{tabular}{lrrrccccc}
\hline & \multicolumn{3}{c}{ NSLN } & & & & \\
OSNA & \multicolumn{1}{c}{+} & - & Total & Sensitivity $^{\mathrm{b}}$ & Specificity $^{\mathrm{b}}$ & PPV $^{\mathrm{b}}$ & \\
\hline+ & 58 & 231 & 289 & $0.906(0.835-0.978)$ & $0.627(0.589-0.665)$ & $0.201(0.155-0.247)$ & $0.985(0.973-0.997)$ \\
- & 6 & 388 & 394 & & & & \\
Total & 64 & 619 & 683 & & & & \\
\hline
\end{tabular}

B, OSNA by NSLN using TTL cut-off of $5,000^{\mathrm{c}}$ copies $/ \mu 1$ for all patients

\begin{tabular}{lccccccc}
\hline & \multicolumn{3}{c}{ NSLN } & & & & \\
nysNA & + & - & Total & Sensitivity & Specificity $^{\mathrm{b}}$ & PPV $^{\mathrm{b}}$ & \\
\hline+ & 50 & 72 & 122 & $0.781(0.680-0.883)$ & $0.884(0.858-0.909)$ & $0.410(0.323-0.497)$ & $0.975(0.962-0.988)$ \\
- & 14 & 547 & 561 & & & & \\
Total & 64 & 619 & 683 & & & & \\
\hline
\end{tabular}

C, OSNA by NSLN using TTL cut-off of $15,000^{\mathrm{c}}$ copies/ $\mu 1$ for all patients

\begin{tabular}{lrrrcccc}
\hline & \multicolumn{3}{c}{ NSLN } & & & \\
OSNA & + & - & Total & Sensitivity $^{\mathrm{b}}$ & Specificity & PPV $^{\mathrm{b}}$ & NPV $^{\mathrm{b}}$ \\
\hline+ & 44 & 58 & 102 & $0.815(0.711-0.918)$ & $0.908(0.885-0.930)$ & $0.431(0.335-0.528)$ & $0.983(0.972-0.992)$ \\
- & 10 & 571 & 581 & & & & \\
Total & 54 & 629 & 683 & & & \\
\hline
\end{tabular}

D, OSNA by NSLN using TTL cut-off of $15,000^{\mathrm{c}}$ copies/ $\mu 1$ for patients undergoing breast conserving surgery

\begin{tabular}{|c|c|c|c|c|c|c|c|}
\hline \multirow[b]{2}{*}{ OSNA } & \multicolumn{3}{|c|}{ NSLN } & \multirow[b]{2}{*}{ Sensitivity $^{\mathrm{b}}$} & \multirow[b]{2}{*}{ Specificity $^{\mathrm{b}}$} & \multirow[b]{2}{*}{$\mathrm{PPV}^{\mathrm{b}}$} & \multirow[b]{2}{*}{$\mathrm{NPV}^{\mathrm{b}}$} \\
\hline & + & - & Total & & & & \\
\hline+ & 20 & 35 & 55 & $0.870(0.732-1.000)$ & $0.927(0.903-0.950)$ & $0.364(0.237-0.491)$ & $0.993(0.986-1.000)$ \\
\hline- & 3 & 441 & 444 & & & & \\
\hline Total & 23 & 476 & 499 & & & & \\
\hline
\end{tabular}

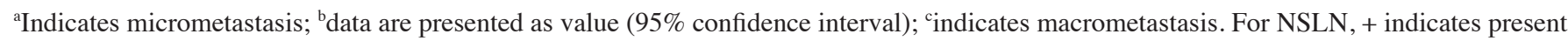
and - indicates absent. For OSNA, + indicates TTL cut-off reached or exceeded and - indicates below TTL cut-off. OSNA, one-step nucleic acid amplification; TTL, total tumour load; NSLN, non-sentinel lymph node; PPV, positive predictive value; NPV, negative predictive value.

Peg et al (7). In the present study, $70.0 \%(14 / 20)$ of patients with a SLN TTL between 5,000 and 15,000 copies/ $\mu$ l had no further metastatic disease identified on ALND. Those patients with a SLN TTL $\geq 15,000$ copies $/ \mu 1$ continue to attract the recommendation to proceed with ALND. In the present cohort, $43.1 \%$ $(44 / 102)$ of these patients $(44 / 683,6.4 \%$ of total cohort) had more than two involved nodes in total. The remaining $56.9 \%(58 / 683$, $8.5 \%$ of total cohort) had only one or two positive nodes, and could be regarded as having their axilla over-treated. In those patients who underwent BCS, 20/23 (87\%) patients who had more than two involved NSLNs on ALND had a SLNB TTL $>15,000$ copies $/ \mu$ l. The $3 / 23(13 \%)$ patients who had more than two nodes involved on ALND following TTL $<15,000$ copies $/ \mu 1$ on OSNA was markedly less than the $23 \%$ of patients estimated to have had residual axillary disease in the non-ALND arm of Z0011, which reported a 0.9 and $1.5 \%$ regional recurrence rate at 5 and 10 years, respectively, for SLN positive disease without ALND $(3,26)$. Our new recommended threshold for no ALND of TTL $<15,000$ copies/ $\mu 1$ of CK19 mRNA is also markedly less than the recently adjusted threshold demonstrated by Peg et al (27) that correlates with disease-free, local recurrence-free and overall survival. They defined a TTL threshold of 25,000 CK19 mRNA copies/ $\mu 1$ for a low-risk group below, and a high-risk group above, this threshold (27). 


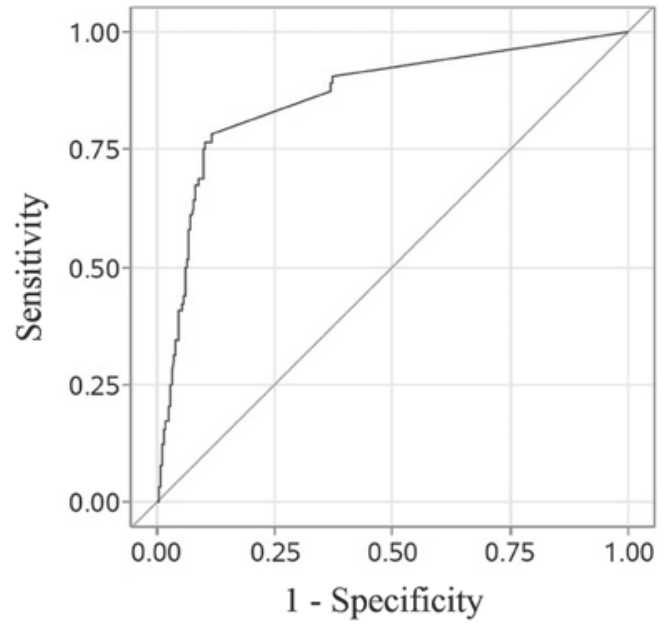

Figure 3. Receiver operating characteristic curve demonstrating diagnostic accuracy. Area under the curve, 0.8618 (95\% confidence interval, 0.8119-0.9118).

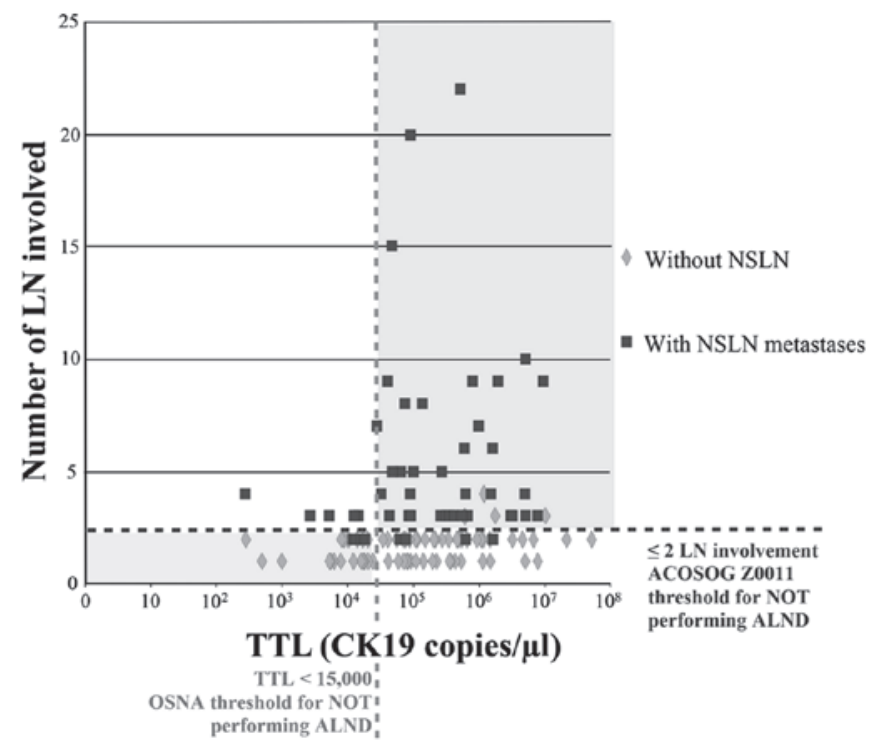

Figure 4. Sentinel lymph node biopsy TTL vs. total lymph node involvement. TTL, total tumour load (of CK19 mRNA copy numbers); LN, lymph node; NSLN, non-sentinel lymph node; ACOSOG Z0011, American College of Surgeons Oncology Group (Alliance) ACOSOG Z0011 randomized trial $(3,26)$; ALND, axillary lymph node dissection; OSNA, one-step nucleic acid cytokeratin-19 amplification assay; CK19, cytokeratin-19.

ALND is not recommended for patients when the SLNB is deemed negative (OSNA TTL $<250$ copies $/ \mu$ l) or interpreted as micrometastatic disease only (OSNA TTL between 250 and $5,000 \mathrm{copies} / \mu \mathrm{l})[25.0 \%(171 / 683)$ in the present cohort] (2). Only $2.3 \%(4 / 171)$ of these patients had more than two involved nodes. We would recommend that patients with a tumour of $<10 \mathrm{~mm}$ diameter receive standard SLNB without OSNA. Only $5.6 \%(8 / 143)$ of these patients had evidence of metastatic axillary disease, none had more than two involved nodes and the rate of involvement was less than the reported 9\% false-negative rate for a two-node assessment (28).

False-negative SLN assessment by OSNA is very low $(1.4 \%)$ and also well within the two SLNB false-negative rate of $9.0 \%$ (29). We suggest that pre-SLNB testing of the tumour

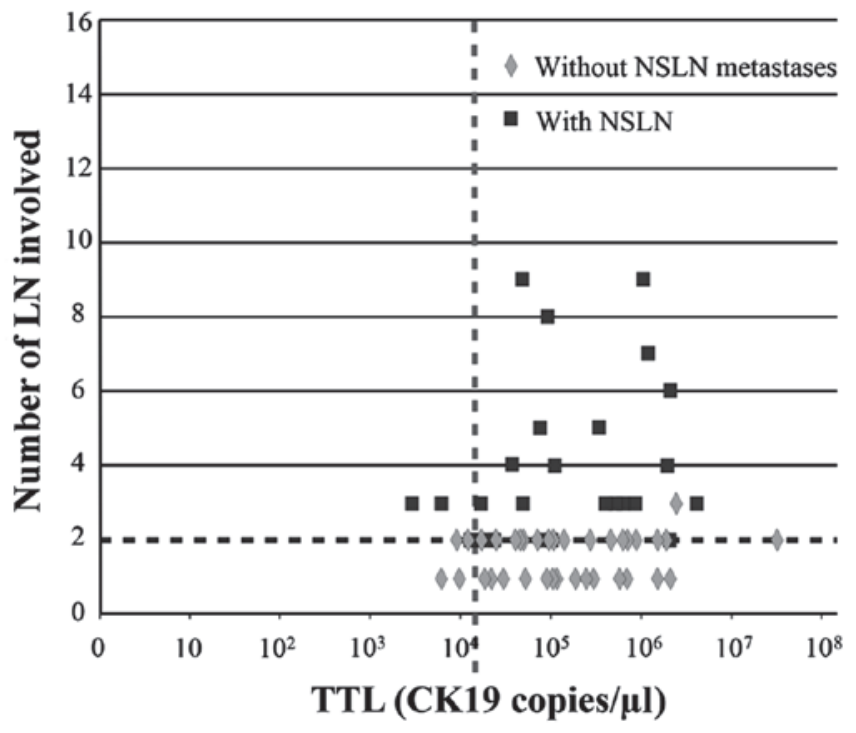

Figure 5. Sentinel lymph node biopsy TTL vs. total lymph node involvement for patients undergoing breast conservation surgery. TTL, total tumour load (of CK19 mRNA copy numbers); LN, lymph node; NSLN, non-sentinel lymph node; CK19, cytokeratin-19.

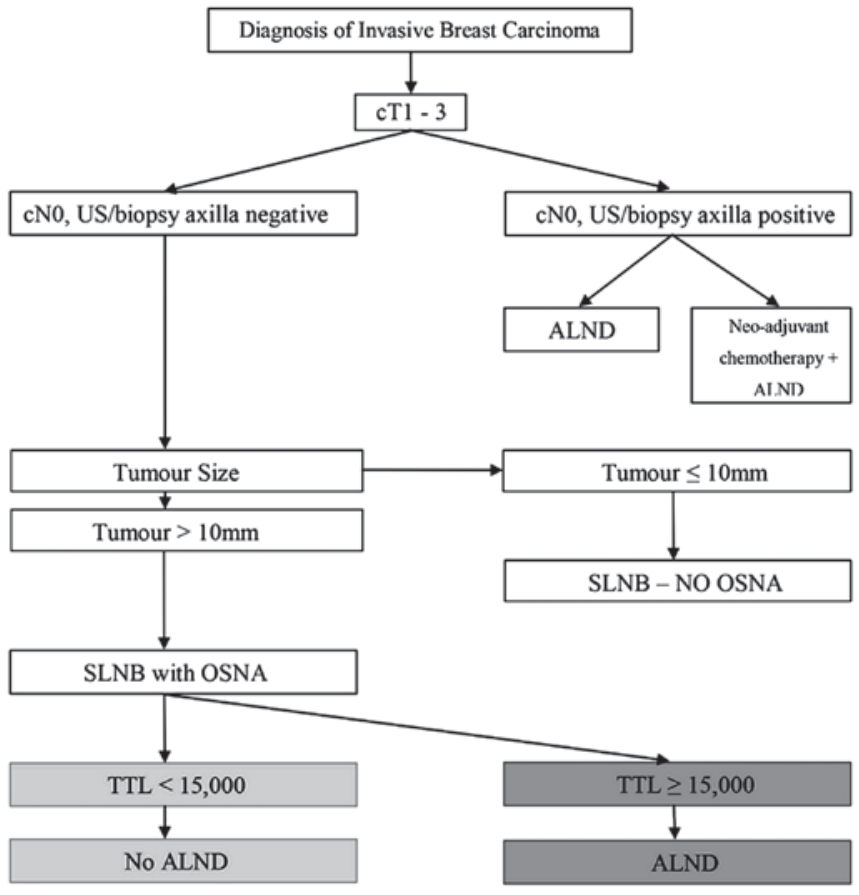

Figure 6. Protocol for management of the clinically uninvolved axilla using OSNA and TTL. OSNA, one-step nucleic acid amplification; TTL, total tumour load (of cytokeratin-19 mRNA copy numbers); cT1-T3 and cN0, TNM classification; US, ultrasound (of axilla); ALND, axillary lymph node dissection; SLNB, sentinel lymph node biopsy.

biopsy specimen with CK19 immunostaining is unnecessary or restricted to defining an inclusion role for OSNA in uncommon circumstances where absent or minimal CK19 mRNA expression may be suspected, such as metaplastic or high Ki67 scoring tumours, inversely reflecting the aggressive nature of the tumour $(29,30)$. Much higher rates of CK19 negative tumours, up to $20 \%$, have been reported; however, these are related to assessments of CK19 immunostaining of protein rather than 
CK19 mRNA levels (30-32). A study by Pegolo et al (33), reported no correlation between CK19 protein expression and CK19 mRNA levels within primary breast cancer or the associated metastatic lymph node. In their study, CK19 mRNA was detected in all cases by OSNA. Similarly, a study by Fujisue et al (29), reported that the incidence of CK19 negative tumours was $12.3 \%$ with immunostaining; however, CK19 mRNA expression was absent in only $1.4 \%$ of the cases (29). These observations have allowed us to generate a protocol for the management of the axilla in our practice (Fig. 6).

The ACOSOG Z0011 trial demonstrated non-inferiority of no ALND for patients with T1 or T2 primary breast lesions and one or two positive axillary lymph nodes having BCS, whole-breast radiotherapy and adjuvant systemic therapy (3). The findings and recommendations of the Z0011 trial have attracted notable controversy and review. These issues have been extensively discussed (34), recently updated (26) and remain defensible in clinical practice. Regardless of controversy, it is clear that not all patients with a positive SLN require an ALND if there is minimal risk of axillary recurrence or compromise of their overall survival. In the present study, 92.1\% of patients had two or less positive nodes on ALND. Axillary recurrence risk is low, even with a reported falsenegative SLNB rate varying between 6.7 and $14.8 \%(1,9,35,36)$. The reported rates of isolated axillary recurrence in SLN negative disease are $\sim 0.6 \%$ at 3 years and $1.1 \%$ at 5 years (37), similar to 0.9 and $1.5 \%$ reported at 5 and 10 years, respectively, for SLN-positive disease without ALND in the Z0011 trial $(3,26)$. It appears illogical to remain preoccupied with the requirement to perform ALND on patients with cT1-T2 disease and a clinically negative axilla when local recurrence is 2.5-11 fold more likely to occur in the fully irradiated breast than the partially irradiated ipsilateral axilla (26). These rates of axillary recurrence are markedly less than the rates of ALND morbidity affecting up to $36 \%$ of patients with lymphoedema (13-19.1\%), paraesthesia (31-37.7\%), pain (21.1\%) and decreased mobility $(11.3 \%)(38,39)$.

In the present study, the results of testing two nodes have provided a robust threshold for clinical decision-making. Four-node testing would potentially alter the management of just $8.5 \%$ (58/683) of the present cohort. Used in combination with TTL, categorical four-node status may facilitate the intraoperative decision to perform ALND along traditional lines, bridging and translating the utility of SLNB TTL in existing models of prognosis and in guiding adjuvant treatment. Enhancement in prediction and treatment guidance is expected with extended genomic biomarker analyses of the OSNA sample homogenate (40).

The observation that $92.1 \%$ of the present total cohort had two or less nodes involved with macrometastatic disease and $63 \%$ of the present patients with positive SLNs did not have additional node metastases supports the move toward selective conservative axillary management. The present study did not demonstrate any benefit in performing ALND on patients with an OSNA TTL $\geq 15,000$ copies $/ \mu 1$. However, while there are no studies that confirm otherwise, it remains practical to identify the ACOSOG two-node macrometastases threshold for guiding clinical practice, informing systemic treatment and axillary radiotherapy field planning, and to putatively improve local disease control in the axilla.
The present study confirmed the thresholds and utility of including OSNA TTL in intraoperative ALND decision modelling that predict and discriminate between $\leq 2$ involved nodes and $>2$ involved nodes. The present study identified, and confirmed, an extended threshold of OSNA TTL that may independently predict when ALND may be avoided, facilitating adoption of the emerging acceptance and recommendations for selective conservative management of the node positive axilla.

\section{References}

1. Krag DN, Anderson SJ, Julian TB, Brown AM, Harlow SP, Costantino JP, Ashikaga T, Weaver DL, Mamounas EP, Jalovec LM, et al: Sentinel-lymph-node resection compared with conventional axillary-lymph-node dissection in clinically node-negative patients with breast cancer: Overall survival findings from the NSABP B-32 randomised phase 3 trial. Lancet Oncol 11: 927-933, 2010.

2. Galimberti V, Cole BF, Zurrida S, Viale G, Luini A, Veronesi P, Baratella P, Chifu C, Sargenti M, Intra M, et al: Axillary dissection versus no axillary dissection in patients with sentinel-node micrometastases (IBCSG 23-01): A phase 3 randomised controlled trial. Lancet Oncol 14: 297-305, 2013.

3. Giuliano AE, Hunt KK, Ballman KV, Beitsch PD, Whitworth PW, Blumencranz PW, Leitch AM, Saha S, McCall LM and Morrow M: Axillary dissection vs no axillary dissection in women with invasive breast cancer and sentinel node metastasis: A randomized clinical trial. JAMA 305: 569-575, 2011.

4. Lyman GH, Temin S, Edge SB, Newman LA, Turner RR, Weaver DL, Benson AB III, Bosserman LD, Burstein HJ, Cody H III, et al: Sentinel lymph node biopsy for patients with early-stage breast cancer: American Society of Clinical Oncology clinical practice guideline update. J Clin Oncol 32: 1365-1383, 2014.

5. Tsujimoto M, Nakabayashi K, Yoshidome K, Kaneko T, Iwase T, Akiyama F, Kato Y, Tsuda H, Ueda S, Sato K, et al: One-step nucleic acid amplification for intraoperative detection of lymph node metastasis in breast cancer patients. Clin Cancer Res 13: 4807-4816, 2007.

6. Di Filippo F, Giannarelli D, Bouteille C, Bernet L, Cano R, Cunnick G and Sapino A: Elaboration of a nomogram to predict non sentinel node status in breast cancer patients with positive sentinel node, intraoperatively assessed with one step nucleic acid amplification method. J Exp Clin Cancer Res 34: 136, 2015.

7. Peg V, Espinosa-Bravo M, Vieites B, Vilardell F, Antúnez JR, de Salas MS, Delgado-Sánchez JJ, Pinto W, Gozalbo F, Petit A, et al: Intraoperative molecular analysis of total tumor load in sentinel lymph node: A new predictor of axillary status in early breast cancer patients. Breast Cancer Res Treat 139: 87-93, 2013.

8. NHS Cancer Screening Programmes/Royal College of Pathologists: Pathology Reporting of Breast Disease. NHSBSP publication no. $58 ; 2005$. Available at: http://www.cancerscreening.nhs.uk/breastscreen/publications/nhsbsp58.html. Accessed May 21, 2016.

9. Mansel RE, MacNeill F, Horgan K, Goyal A, Britten A, Townson J, Clarke D, Newcombe RG, Keshtgar M, Guildford Breast Surgeons, et al: Results of a national training programme in sentinel lymph node biopsy for breast cancer. Br J Surg 100: 654-661, 2013.

10. Koca B, Kuru B, Ozen N, Yoruker S and Bek Y: A breast cancer nomogram for prediction of non-sentinel node metastasis-validation of fourteen existing models. Asian Pac J Cancer Prev 15: 1481-1488, 2014.

11. Glechner A, Wöckel A, Gartlehner G, Thaler K, Strobelberger M, Griebler U and Kreienberg R: Sentinel lymph node dissection only versus complete axillary lymph node dissection in early invasive breast cancer: A systematic review and meta-analysis. Eur J Cancer 49: 812-825, 2013.

12. Straver ME, Meijnen P, van Tienhoven G, van de Velde CJ, Mansel RE, Bogaerts J, Demonty G, Duez N, Cataliotti L, Klinkenbijl J, et al: Role of axillary clearance after a tumor-positive sentinel node in the administration of adjuvant therapy in early breast cancer. J Clin Oncol 28: 731-737, 2010. 
13. Nadeem RM, Gudur LD and Saidan ZA: An independent assessment of the 7 nomograms for predicting the probability of additional axillary nodal metastases after positive sentinel lymph node biopsy in a cohort of British patients with breast cancer. Clin Breast Cancer 14: 272-279, 2014.

14. Meretoja TJ, Audisio RA, Heikkilä PS, Bori R, Sejben I, Regitnig P, Luschin-Ebengreuth G, Zgajnar J, Perhavec A, Gazic B, et al: International multicenter tool to predict the risk of four or more tumour-positive axillary lymph nodes in breast cancer patients with sentinel node macrometastases. Breast Cancer Res Treat 138: 817-827, 2013.

15. Katz A, Smith BL, Golshan M, Niemierko A, Kobayashi W, Raad RA, Kelada A, Rizk L, Wong JS, Bellon JR, et al: Nomogram for the prediction of having four or more involved nodes for sentinel lymph node-positive breast cancer. J Clin Oncol 26: 2093-2098, 2008.

16. Chagpar AB, Scoggins CR, Martin RC II, Cook EF, McCurry T, Mizuguchi N, Paris KJ, Carlson DJ, Laidley AL, El-Eid SE, et al: Predicting patients at low probability of requiring post-mastectomy radiation therapy. Ann Surg Oncol 14: 670-677, 2007.

17. Rivers AK, Griffith KA, Hunt KK, Degnim AC, Sabel MS, Diehl KM, Cimmino VM, Chang AE, Lucas PC and Newman LA: Clinicopathologic features associated with having four or more metastatic axillary nodes in breast cancer patients with a positive sentinel lymph node. Ann Surg Oncol 13: 36-44, 2006.

18. Kubota M, Komoike Y, Hamada M, Shinzaki W, Azumi T, Hashimoto Y, Imoto S, Takeyama Y and Okuno K: One-step nucleic acid amplification assay for intraoperative prediction of advanced axillary lymph node metastases in breast cancer patients with sentinel node metastasis. Mol Clin Oncol 4: $173-178,2016$.

19. Piñero-Madrona A, Ruiz-Merino G,BernetL,Miguel-Martínez B, Vicente-García F, Viguri-Díaz MA and Giménez-Climent J: Tumoral load quantification of positive sentinel lymph nodes in breast cancer to predict more than two involved nodes. Breast 23 : $859-864,2014$

20. Intraoperative tests (RD-100i OSNA system and Metasin test) for detecting sentinel lymph node metastases in breast cancer. NICE diagnostics guidance 8. August 2013. Available at: http://www. nice.org.uk/dg8. Accessed May 21, 2016.

21. Raia-Barjat T, Trombert B, Khaddage A, Douchet C, Seffert P, Peoc'h M, Falk AT, Magné N and Chauleur C: OSNA (one-step nucleic acid amplification) sentinel lymph node intraoperative molecular analysis in breast cancer: A cost-benefit analysis. Med Oncol 31: 322, 2014

22. Brambilla T, Fiamengo B, Tinterri C, Testori A, Grassi MM, Sciarra A, Abbate T, Gatzemeier W, Roncalli $M$ and Di Tommaso L: One-step nucleic acid amplification in breast cancer sentinel lymph node: A single institutional experience and a short review. Front Med (Lausanne) 2: 37, 2015.

23. Espinosa-Bravo M, Sansano I, Pérez-Hoyos S, Ramos M, Sancho M, Xercavins J, Rubio IT and Peg V: Prediction of non-sentinel lymph node metastasis in early breast cancer by assessing total tumoral load in the sentinel lymph node by molecular assay. Eur J Surg Oncol 39: 766-773, 2013.

24. Ohi Y, Umekita Y, Sagara Y, Rai Y, Yotsumoto D, Matsukata A, Baba S, Tamada S, Matsuyama Y, Ando M, et al: Whole sentinel lymph node analysis by a molecular assay predicts axillary node status in breast cancer. Br J Cancer 107: 1239-1243, 2012

25. Osako T, Iwase T, Kimura K, Horii R and Akiyama F: Sentinel node tumour burden quantified based on cytokeratin 19 mRNA copy number predicts non-sentinel node metastases in breast cancer: Molecular whole-node analysis of all removed nodes. Eur J Cancer 49: 1187-1195, 2013.

26. Giuliano A, Ballman K, McCall L, Beitsch P, Whitworth PW, Blumencranz P, Leitch AM, Saha S, Morrow M and Hunt KK: Locoregional recurrence after sentinel lymph node dissection with or without axillary dissection in patients with sentinel lymph node metastases: Long-term Follow-up From the American College of Surgeons Oncology Group (Alliance) ACOSOG Z0011 Randomized Trial. Ann Surg 264: 413-420, 2016.
27. Peg V, Sansano I, Vieites B, Bernet L, Cano R, Córdoba A, Sancho M, Martín MD, Vilardell F, Cazorla A, et al: Role of total tumour load of sentinel lymph node on survival in early breast cancer patients. Breast 33: 8-13, 2017.

28. Goyal A, Newcombe RG and Mansel RE; Axillary Lymphatic Mapping Against Nodal Axillary Clearance (ALMANAC) Trialists Group: Clinical relevance of multiple sentinel nodes in patients with breast cancer. Br J Surg 92: 438-442, 2005.

29. Fujisue M, Nishimura R, Okumura Y, Tashima R, Nishiyama Y, Osako T, Toyozumi Y and Arima N: Clinical significance of CK19 negative breast cancer. Cancers (Basel) 5: 1-11, 2012.

30. Vilardell F, Novell A, Martin J, Santacana M, Velasco A, Díez-Castro MJ, Cuevas D, Panadés MJ, González S, Llombart A, et al: Importance of assessing CK19 immunostaining in core biopsies in patients subjected to sentinel node study by OSNA. Virchows Arch 460: 569-575, 2012.

31. Tamaki Y: One-step nucleic acid amplification assay (OSNA) for sentinel lymph node biopsy. Breast Cancer 22: 230-234, 2015.

32. Alvarenga CA, Paravidino PI, Alvarenga M, Dufloth R, Gomes M, Zeferino LC and Schmitt F: Expression of CK19 in invasive breast carcinomas of special histological types: Implications for the use of one-step nucleic acid amplification. J Clin Pathol 64: 493-497, 2011.

33. Pegolo E, Puppin C, Gerometta A, Damante G, Puglisi F and Di Loreto C: One-step nucleic acid amplification (OSNA) for intraoperative evaluation of sentinel lymph node status in breast cancer: A comparative study between CK19 protein expression and CK19 mRNA level in primary tumors and lymph node metastasis. Virchows Arch 463: 7-15, 2013.

34. Shah-Khan M and Boughey JC: Evolution of axillary node staging in breast cancer: Clinical implications of the ACOSOG Z0011 trial. Cancer Control 19: 267-276, 2012.

35. Kohrt HE, Olshen RA, Bermas HR, Goodson WH, Wood DJ, Henry S, Rouse RV, Bailey L, Philben VJ, Dirbas FM, et al: New models and online calculator for predicting non-sentinel lymph node status in sentinel node positive breast cancer patients. BMC Cancer 8: 66, 2008.

36. Goyal A, Newcombe RG, Chhabra A and Mansel RE; ALMANAC Trialists Group: Factors affecting failed localisation and false-negative rates of sentinel node biopsy in breast cancer--results of the ALMANAC validation phase. Breast Cancer Res Treat 99: 203-208, 2006.

37. Bergkvist L, de Boniface J, Jönsson PE, Ingvar C, Liljegren G and Frisell J; Swedish Society of Breast Surgeons: Axillary recurrence rate after negative sentinel node biopsy in breast cancer: Three year follow-up of the Swedish multicenter cohort study. Ann Surg 247: 150-156, 2008.

38. Mansel RE, Fallowfield L, Kissin M, Goyal A, Newcombe RG, Dixon JM, Yiangou C, Horgan K, Bundred N, Monypenny I, et al: Randomized multicenter trial of sentinel node biopsy versus standard axillary treatment in operable breast cancer: The ALMANAC trial. J Natl Cancer Inst 98: 599-609, 2006

39. Langer I, Guller U, Berclaz G, Koechli OR, Schaer G, Fehr MK, Hess T, Oertli D, Bronz L, Schnarwyler B, et al: Morbidity of sentinel lymph node biopsy (SLN) alone versus SLN and completion axillary lymph node dissection after breast cancer surgery: A prospective Swiss multicenter study on 659 patients. Ann Surg 245: 452-461, 2007.

40. Martín-Sánchez E, Pernaut-Leza E, Mendaza S, Cordoba A, Vicente-Garcia F, Monreal-Santesteban I, Vizcaino JP, De Cerio MJ, Perez-Janices N, Blanco-Luquin I, et al: Gene promoter hypermethylation is found in sentinel lymph nodes of breast cancer patients, in samples identified as positive by one-step nucleic acid amplification of cytokeratin 19 mRNA. Virchows Arch 469: 51-59, 2016. 